\section{A Sex Difference in HandScan Scores in Rheumatoid Arthritis Patients and Controls? An Ongoing Analysis of the Sex Difference and Other Potential Confounders}

\section{To the Editor:}

A recent paper by Triantafyllias, et al described that optical spectral transmission (OST) scores, obtained by the HandScan, were significantly higher in male compared to female patients with rheumatoid arthritis (RA) and controls, and an association between OST score and age, BMI, and hand surface area was found. ${ }^{1}$

The difference in sex that Triantafyllias, et al showed is in line with results of studies performed at our department. We developed and validated a new disease activity index, Disease Activity Score (DAS)-OST, in which tender and swollen joint counts are replaced by the OST score. In the construction of the DAS-OST formula, sex had to be taken into account, because in this study, OST scores were higher in males than in females. ${ }^{2}$ In addition, by comparing single OST scores to the rheumatologist's clinical classification into active or inactive RA as reference, different optimal cutoffs for males and females were calculated, again indicating a sex difference.

Based on these findings, we investigated whether this difference in sex was also observed in controls ( 30 males vs 45 females). This indeed turned out to be the case $(P=0.02)$, and is in line with the results of Triantafyllias, et al. ${ }^{1}$ Figure 1 shows the OST scores in patients with RA and controls, separated according to sex, indicating the sex difference, as well as the difference between patients with RA (generally low disease activity) and controls; however, there are considerable overlaps in scores between the 4 subgroups.
Further, we aimed to quantify the OST score differences between males and females in patients with RA as well as in controls, and to determine which factors influence OST scores, including sex. In total, 77 participants were included in this study. In this whole sample, adjusting for cohort (RA vs controls), no statistically significant difference in sex was found $(P=0.12)$. However, in the subgroup of controls (i.e., 22 females vs 15 males), a statistically significant difference in OST score was observed $(P=0.05)$, in contrast to the subgroup of patients with RA ( $P=0.90,20$ females vs 20 males). A possible explanation for the latter result, and for the absence of a statistically significant sex difference when analyzing the whole sample, could be a difference in disease activity between male and female patients with RA. Unfortunately, we were not able to adjust for this, as disease activity data were not available. For OST scores in the male subgroup, predictors were cohort, i.e., RA patients vs controls (estimate, i.e., mean difference in OST score, $-3.08,95 \% \mathrm{CI}-6.52$ to 0.36 , indicating lower OST scores in controls), age (estimate $-0.15,95 \% \mathrm{CI}-0.29$ to -0.02 , indicating lower OST scores in older persons), and hand surface area (estimate $0.24,95 \% \mathrm{CI}$ $0.03-0.45$, indicating higher OST scores with larger hand surface area). For OST scores in the female subgroup, these were cohort (estimate $-4.33,95 \%$ CI -7.17 to -1.49 , indicating lower OST scores in controls), BMI (estimate $-0.44,95 \%$ CI -0.79 to -0.09 , indicating lower OST scores with higher $\mathrm{BMI}$ ), and hand volume (estimate 0.03 , 95\% CI $0.01-0.05$, indicating higher OST scores with more hand volume). In our opinion, not all of these results are easy to explain.

To exclude a system error in the HandScan device, despite manufacturer assurances that sex is not a factor in the algorithm for calculating the OST score, we aimed to perform an additional study in which a HandScan measurement is performed twice within the same participant; it is already known that the test-retest reliability, based on 2 measurements, is good on the patient level. ${ }^{3}$ However, due to the COVID-19 (coronavirus disease

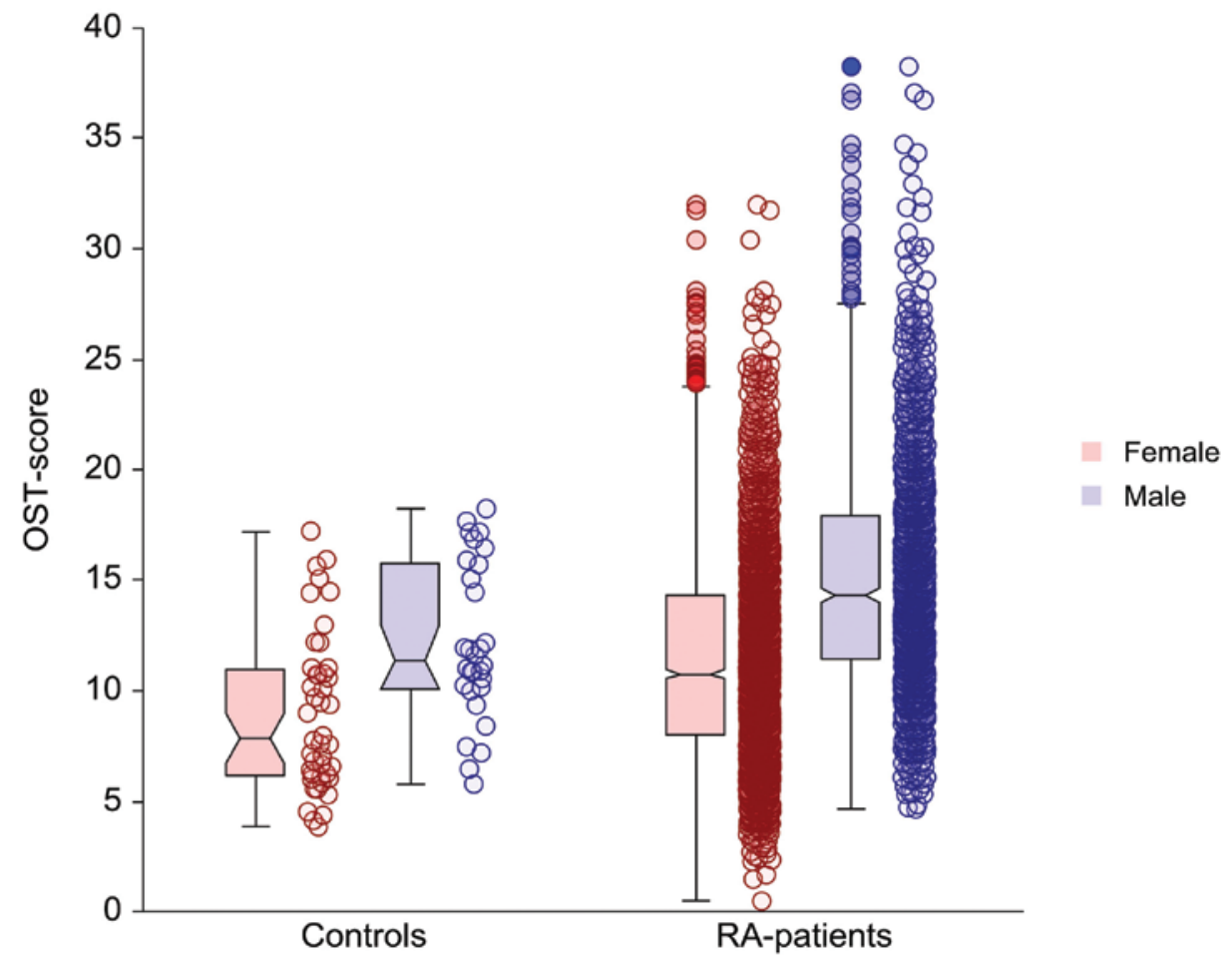

Figure 1. Figure distribution of OST scores in subgroups; there is a single score for each individual. Controls: females $(\mathrm{n}=45)$, males $(\mathrm{n}=30)$; females with RA $(\mathrm{n}=2200)$; males with RA $(\mathrm{n}=1157)$. OST: optical spectral transmission, score range 0-66 (worst); RA: rheumatoid arthritis. 
2019; caused by SARS-CoV-2) pandemic, it was not feasible to perform this study yet.

In summary, OST scores in controls and probably also in patients with RA are statistically significantly higher in males compared to females, although there is considerable overlap in OST scores between subgroups. This suggests that the HandScan cannot be used for the diagnosis of RA and has drawbacks when comparing disease activity between groups of female and male RA patients in research. The gender difference, which has to be analyzed further, plays no role, however, when repeated OST scores, whether or not integrated in an index, are used in individual RA patients, to evaluate in clinical practice their disease activity over time.,

Maxime M.A. Verhoeven ${ }^{1}\left(\mathbb{D}, \mathrm{MSc}_{\mathrm{c}}\right.$

Antonius A.A. Westgeest ${ }^{2}, \mathrm{MD}, \mathrm{PhD}$

Johannes W.G. Jacobs ${ }^{1} \mathbb{D}, \mathrm{MD}, \mathrm{PhD}$

${ }^{1}$ Department of Rheumatology and Clinical Immunology, University

Medical Center Utrecht, Utrecht University, Utrecht;

${ }^{2}$ Department of Rheumatology, Máxima MC, Eindhoven, the Netherlands. The authors have no conflicts of interest.

The institutional ethical review board of Máxima MC confirmed that the Medical Research Involving Human Subjects Act (WMO) was not applicable (N19.002, N19.122-L19.137, N20.056-L20.074). In this case, no interventions or extra measurements were performed and only pseudonymized routinely collected data were extracted from medical records.
As such, patients did not give informed consent. If data were collected on controls or extra measurements were performed on patients with RA to collect data, all participants gave their informed consent.

Address correspondence to Dr. M.M. Verhoeven, Department of Rheumatology \& Clinical Immunology G02.228,

P.O. Box 85500 , 3508GA Utrecht, the Netherlands.

Email:m.m.a.verhoeven-15@umcutrecht.nl.

\section{REFERENCES}

1. Triantafyllias K, Heller C, de Blasi M, Galle P, Schwarting A. Diagnostic value of optical spectral transmission in rheumatoid arthritis: associations with clinical characteristics and comparison with joint ultrasonography. J Rheumatol 2020;47:1314-22.

2. Verhoeven MM, Welsing PM, Tekstra J, van Laar JM, Lafeber FP, Westgeest AA. Development of a disease activity index for RA patients using HandScan. Ann Rheum Dis 2020;79:879.

3. Besselink NJ, van der Meijde P, Rensen WH, Meijer PB, Marijnissen AC, van Laar JM, et al. Optical spectral transmission to assess inflammation in hand and wrist joints of rheumatoid arthritis patients. Rheumatology 2018;57:865-72.

4. Verhoeven MM, Tekstra J, Marijnissen AC, Meier AJ, Westgeest AA, Lafeber FP, et al. Utility of the HandScan in monitoring disease activity and prediction of clinical response in rheumatoid arthritis patients. Rheumatol Adv Pract 2021;5:rkab004. 